\title{
MODELING OF SHOCK-WAVE PROCESSES IN ALUMINUM UNDER THE ACTION OF A SHORT LASER PULSE
}

\author{
A. YU. SEMENOV ${ }^{1,2}$, I. A. STUCHEBRYUKHOV ${ }^{1}$ AND K. V. KHISHCHENKO ${ }^{3,2,4^{*}}$ \\ ${ }^{1}$ Prokhorov General Physics Institute of the Russian Academy of Sciences \\ Vavilova 38, 119991 Moscow, Russia \\ 2 Moscow Institute of Physics and Technology \\ Institutskiy Pereulok 9, 141701 Dolgoprudny, Moscow Region, Russia \\ ${ }^{3}$ Joint Institute for High Temperatures of the Russian Academy of Sciences \\ Izhorskaya 13 Bldg 2, 125412 Moscow, Russia \\ ${ }^{4}$ South Ural State University \\ Lenin Avenue 76, 454080 Chelyabinsk, Russia \\ *Corresponding author. E-mail: konst@ihed.ras.ru
}

\section{DOI: 10.20948/mathmontis-2021-50-10}

Summary. A hydrodynamic model of shock-wave processes in a material under the action of a short high-intensity laser pulse is considered. The simulation is carried out for the case of an aluminum target $90 \mu \mathrm{m}$ thick, irradiated by a laser pulse with a duration of $70 \mathrm{ps}$ and a maximum intensity of $14.7 \mathrm{TW} / \mathrm{cm}^{2}$. In the corresponding laboratory experiment, on the rear side of the target after irradiation, a spall of a part of the material is recorded at a depth of $10 \pm 1 \mu \mathrm{m}$. Calculation of the time dependence of the pressure and density of aluminum in the spall plane makes it possible to determine the tensile strength of aluminum at a high strain rate.

\section{INTRODUCTION}

The action of a short high-intensity laser pulse upon a target makes it possible to study the properties of the target material under shock-wave loading at a high strain rate [1-9]. Numerical modeling of such a process $[2,3,6,10-16]$ provides additional possibilities for the interpretation of the obtained measurement results.

In this work, an example of a laboratory experiment on the action of a 70 ps laser pulse on an aluminum target is given. A description of the hydrodynamic model for the propagation of shock compression waves and adiabatic unloading along the target is presented. The results of modeling are presented and a conclusion is made about the magnitude of the spall strength of aluminum at the considered strain rate. 


\section{EXPERIMENT}

The experiment was carried out on a Kamerton-T facility based on a neodymium glass laser (wavelength $\lambda=0.527 \mu \mathrm{m}$ ) $[8,10,11]$. A pulse with duration $\tau=70 \mathrm{ps}$ and energy $E_{1}=$ $3.57 \mathrm{~J}$ was focused into a spot $0.63 \mathrm{~mm}$ in diameter on the surface of a $90-\mu \mathrm{m}$-thick aluminum target. Taking into account the measured dependence of the laser radiation intensity on time, the maximum intensity of this pulse is estimated to be $I_{0}=14.7 \mathrm{TW} / \mathrm{cm}^{2}$.

The result of the action of such a pulse is the formation of a spall of a part of the material on the rear side of the target. The spall occurred at a distance of $10 \pm 1 \mu \mathrm{m}$ from the rear surface; the diameter of the spalled plate is $0.66 \mathrm{~mm}$.

\section{HYDRODYNAMIC MODEL}

The system of hydrodynamic equations for the one-dimensional case under consideration has the following form [17]:

$$
\begin{gathered}
\frac{\partial}{\partial t} \mathbf{U}+\frac{\partial}{\partial x} \mathbf{F}=0, \\
\mathbf{U}=\left(\begin{array}{c}
\rho \\
\rho u \\
\rho v \\
\rho w \\
e
\end{array}\right), \quad \mathbf{F}=\left(\begin{array}{c}
\rho u \\
\rho u^{2}+P \\
\rho u v \\
\rho u w \\
(e+P) u
\end{array}\right),
\end{gathered}
$$

where $t$ is the time coordinate; $x$ is the spatial coordinate; $\rho$ is the density of the material under consideration; $P$ is the pressure; $e$ is the full energy density,

$$
e=\rho E+\frac{1}{2} \rho\left(u^{2}+v^{2}+w^{2}\right)
$$

$E$ is the specific internal energy; $u$ is the particle velocity along the $x$-axis; $v=0$ and $w=0$ for the case.

In quasilinear non-conservative form, the system of equations (1) can be written as follows:

$$
\frac{\partial}{\partial t} \mathbf{U}+\mathbf{A} \frac{\partial}{\partial x} \mathbf{U}=0
$$


where

$$
\begin{aligned}
& \mathbf{A}=\left(\begin{array}{ccccc}
0 & 1 & 0 & 0 & 0 \\
-u^{2}+\theta b & 2 u-u b & -v b & -w b & b \\
-u v & v & u & 0 & 0 \\
-u w & w & 0 & u & 0 \\
-u h+u \theta b & h-u^{2} b & -u v b & -u w b & u+u b
\end{array}\right) \\
& h=\frac{e+P}{\rho}, \quad \theta=q^{2}-\frac{e}{\rho}+\frac{(\partial P / \partial \rho)_{E}}{b}, \quad q^{2}=u^{2}+v^{2}+w^{2}, \quad b=\frac{(\partial P / \partial E)_{\rho}}{\rho} \text {. }
\end{aligned}
$$

One can write matrix $\mathbf{A}$ in the form $\mathbf{A}=\Omega \Lambda \Omega^{-1}$, where

$$
\begin{gathered}
\Omega=\left(\begin{array}{ccccc}
1 & 0 & 0 & 1 & 1 \\
u-c & 0 & 0 & u & u+c \\
v & 1 & 0 & v & v \\
w & 0 & 1 & w & w \\
h-u c & v & w & h-c^{2} b^{-1} & h+u c
\end{array}\right), \quad \Lambda=\left(\begin{array}{ccccc}
u-c & 0 & 0 & 0 & 0 \\
0 & u & 0 & 0 & 0 \\
0 & 0 & u & 0 & 0 \\
0 & 0 & 0 & u & 0 \\
0 & 0 & 0 & 0 & u+c
\end{array}\right), \\
\Omega^{-1}=\frac{b}{2 c^{2}}\left(\begin{array}{ccccc}
\theta+u c b^{-1} & -u-c b^{-1} & -v & -w & 1 \\
-2 v c^{2} b^{-1} & 0 & 2 c^{2} b^{-1} & 0 & 0 \\
-2 w c^{2} b^{-1} & 0 & 0 & 2 c^{2} b^{-1} & 0 \\
2 h-2 q^{2} & 2 u & 2 v & 2 w & -2 \\
\theta-u c b^{-1} & -u+c b^{-1} & -v & -w & 1
\end{array}\right) \\
\operatorname{det} \Omega=\frac{2 c^{3}, \quad c=\sqrt{(\partial P / \partial \rho)_{E}+\frac{P}{\rho^{2}}(\partial P / \partial E)_{\rho}}}{b},
\end{gathered}
$$

Here, $c$ is the adiabatic sound velocity. Using values $c$ and $h$, one can obtain

$$
\theta=q^{2}-h+c^{2} b^{-1}
$$

The system of equations of motion (1) is closed by the equation of state of the target material in the form of a function

$$
P=P(\rho, E),
$$

which is taken according to the model [18-20]. 


\section{SOLUTION METHOD}

With the use of the formulas for matrices from the previous section, the system of equations (1) and (11) can be solved by the Courant-Isaacson-Rees method [21]. The difference scheme of the method is as follows [17]:

$$
\begin{gathered}
\frac{\mathbf{U}_{j}^{k+1}-\mathbf{U}_{j}^{k}}{\Delta t}+\frac{\mathbf{F}_{j+1 / 2}-\mathbf{F}_{j-1 / 2}}{\Delta x}=0 \\
\mathbf{F}_{m+1 / 2}=\frac{1}{2}\left(\mathbf{F}_{m}^{k}+\mathbf{F}_{m+1}^{k}\right)+\frac{1}{2}|\mathbf{A}|_{m+1 / 2}^{k}\left(\mathbf{U}_{m}^{k}-\mathbf{U}_{m+1}^{k}\right)
\end{gathered}
$$

for $m=j-1$ and $j$. Here, integer subscripts denote the values of the function at the centers of discrete grid cells in space, and half-integer ones-at the boundaries of the cells; $\Delta x$ is the step of a uniform grid in space; $\Delta t$ is the time step;

$$
|\Lambda|=\left(\begin{array}{ccccc}
|u-c| & 0 & 0 & 0 & 0 \\
0 & |u| & 0 & 0 & 0 \\
0 & 0 & |u| & 0 & 0 \\
0 & 0 & 0 & |u| & 0 \\
0 & 0 & 0 & 0 & |u+c|
\end{array}\right) .
$$

Matrix (15) can be represented as a sum of three matrices with multipliers:

$$
|\Lambda|=|u|\left(\begin{array}{ccccc}
1 & 0 & 0 & 0 & 0 \\
0 & 1 & 0 & 0 & 0 \\
0 & 0 & 1 & 0 & 0 \\
0 & 0 & 0 & 1 & 0 \\
0 & 0 & 0 & 0 & 1
\end{array}\right)+\alpha\left(\begin{array}{ccccc}
1 & 0 & 0 & 0 & 0 \\
0 & 0 & 0 & 0 & 0 \\
0 & 0 & 0 & 0 & 0 \\
0 & 0 & 0 & 0 & 0 \\
0 & 0 & 0 & 0 & 0
\end{array}\right)+\gamma\left(\begin{array}{ccccc}
0 & 0 & 0 & 0 & 0 \\
0 & 0 & 0 & 0 & 0 \\
0 & 0 & 0 & 0 & 0 \\
0 & 0 & 0 & 0 & 0 \\
0 & 0 & 0 & 0 & 1
\end{array}\right),
$$

where $\alpha=|u-c|-|u| ; \gamma=|u+c|-|u|$.

Denoting $\Delta \mathbf{U}=\mathbf{U}_{m}-\mathbf{U}_{m+1}$ (with elements $\Delta U=U_{m}-U_{m+1}$ ) and using (14) and (16), one can obtain

$$
|\mathbf{A}| \Delta \mathbf{U}=|u|\left(\begin{array}{c}
\Delta \rho \\
\Delta(\rho u) \\
\Delta(\rho v) \\
\Delta(\rho w) \\
\Delta e
\end{array}\right)+\alpha(f+g)\left(\begin{array}{c}
1 \\
u-c \\
v \\
w \\
h-u c
\end{array}\right)+\gamma(f-g)\left(\begin{array}{c}
1 \\
u+c \\
v \\
w \\
h+u c
\end{array}\right)
$$


where

$$
f=\frac{b}{2 c^{2}}[\theta \Delta \rho-u \Delta(\rho u)-v \Delta(\rho v)-w \Delta(\rho w)+\Delta e], \quad g=\frac{1}{2 c}[u \Delta \rho-\Delta(\rho u)] .
$$

Taking into account relations (6) as well as $\Delta P \approx(\partial P / \partial \rho)_{E} \Delta \rho+(\partial P / \partial E)_{\rho} \Delta E$ and $\Delta(\rho u) \approx$ $\rho \Delta u+u \Delta \rho$, it is possible to obtain approximate expressions for the factors $f$ and $g$ :

$$
f \approx \frac{1}{2 c^{2}} \Delta P, \quad g \approx-\frac{\rho}{2 c} \Delta u
$$

So, in (13), one can use

$$
\begin{gathered}
|\mathbf{A}|_{m+1 / 2}^{k}\left(\mathbf{U}_{m}^{k}-\mathbf{U}_{m+1}^{k}\right)=\left(\begin{array}{c}
|u|_{m+1 / 2}^{k}\left(\rho_{m}^{k}-\rho_{m+1}^{k}\right)+\beta \beta_{m+1 / 2}^{k} \\
|u|_{m+1 / 2}^{k}\left([\rho u]_{m}^{k}-[\rho u]_{m+1}^{k}\right)+[\beta u-\delta c]_{m+1 / 2}^{k} \\
|u|_{m+1 / 2}^{k}\left([\rho v]_{m}^{k}-[\rho v]_{m+1}^{k}\right)+[\beta v]_{m+1 / 2}^{k} \\
|u|_{m+1 / 2}^{k}\left([\rho w]_{m}^{k}-[\rho w]_{m+1}^{k}\right)+[\beta w]_{m+1 / 2}^{k} \\
|u|_{m+1 / 2}^{k}\left(e_{m}^{k}-e_{m+1}^{k}\right)+[\beta h-\delta u c]_{m+1 / 2}^{k}
\end{array}\right), \\
\beta_{m+1 / 2}^{k}=[\alpha(f+g)+\gamma(f-g)]_{m+1 / 2}^{k}, \quad \delta_{m+1 / 2}^{k}=[\alpha(f+g)-\gamma(f-g)]_{m+1 / 2}^{k}, \\
f_{m+1 / 2}^{k}=\left[\frac{1}{2 c^{2}}\right]_{m+1 / 2}^{k}\left(P_{m}^{k}-P_{m+1}^{k}\right), \quad g_{m+1 / 2}^{k}=-\left[\frac{\rho}{2 c}\right]_{m+1 / 2}^{k}\left(u_{m}^{k}-u_{m+1}^{k}\right) .
\end{gathered}
$$

At the initial moment of time, the entire target was divided in thickness into cells of the same size, which were numbered from $j=1$ to $N_{j}=1000$. This gives the step of the grid in space $\Delta x=0.09 \mu \mathrm{m}$. The step in time was chosen from the condition $\Delta t \leqslant \xi \Delta x / \max (|u|+c)$, where $\xi=0.1$.

At each time step, the boundaries of the cells shifted with a certain velocity $D$, which is the particle velocity $u$ for the case under consideration:

$$
x_{m+1 / 2}^{k+1}=x_{m+1 / 2}^{k}+D_{m+1 / 2}^{k} \Delta t .
$$

The construction of a difference scheme for such a moving grid is based upon the system of hydrodynamic equations in integral form:

$$
\oint_{L}(\mathbf{U d} x-\mathbf{F} \mathrm{d} t)=0
$$

where $L$ is the contour that bounds the region of integration on the coordinate plane $(x, t)$. As this contour $L$, it is suitable to take a difference cell with number $j$ with height $\Delta t$ and bases $\Delta x_{j}^{k+1}$ and $\Delta x_{j}^{k}$, where $\Delta x_{m}=x_{m+1 / 2}-x_{m-1 / 2}$. Approximating integral equation (24), one can 
obtain

$$
(\mathbf{U} \Delta x)_{j}^{k+1}-(\mathbf{U} \Delta x)_{j}^{k}+\mathbf{F}_{j+1} \Delta t-\mathbf{F}_{j-1} \Delta t=0,
$$

or, instead of (12),

$$
\frac{(\mathbf{U} \Delta x)_{j}^{k+1}-(\mathbf{U} \Delta x)_{j}^{k}}{\Delta t}+\mathbf{F}_{j+1}-\mathbf{F}_{j-1}=0
$$

A local transition to a coordinate system that moves with constant velocity $D$ relative to the original system (Galilean transformation) changes the original form of the system of equations (1):

$$
\frac{\partial}{\partial t} \mathbf{U}+\frac{\partial}{\partial x}(\mathbf{F}-D \mathbf{U})=0
$$

In this regard, the flows (13) at the boundaries of the cells change:

$$
\begin{gathered}
\mathbf{F}_{m+1 / 2}=(\mathbf{F}-D \mathbf{U})_{m+1 / 2} \\
=\frac{1}{2}\left(\mathbf{F}_{m}^{k}+\mathbf{F}_{m+1}^{k}\right)-\frac{1}{2}\left(\mathbf{U}_{m}^{k}+\mathbf{U}_{m+1}^{k}\right) D_{m+1 / 2}^{k}+\frac{1}{2}\left|\mathbf{A}_{D}\right|_{m+1 / 2}^{k}\left(\mathbf{U}_{m}^{k}-\mathbf{U}_{m+1}^{k}\right), \\
\left|\Lambda_{D}\right|=\left(\begin{array}{ccccc}
|u-D-c|=\Omega\left|\Lambda_{D}\right| \Omega^{-1}, \\
0 & 0 & 0 & 0 & 0 \\
0 & 0 & |u-D| & 0 & 0 \\
0 & 0 & 0 & |u-D| & 0 \\
0 & 0 & 0 & 0 & |u-D+c|
\end{array}\right) .
\end{gathered}
$$

\section{INITIAL CONDITIONS}

The initial values of pressure, density and particle velocity were set constant over the target: $P=0.1 \mathrm{MPa}, \rho=\rho_{0}=2.712 \mathrm{~g} / \mathrm{cm}^{3}$ and $u=v=w=0$. The initial value of specific internal energy was taken according to the used equation of state for aluminum.

\section{BOUNDARY CONDITIONS}

On the irradiated surface of the target, a pressure profile

$$
P(t)=P_{\mathrm{a}} 16^{-\left(t-t_{0}\right)^{2} \tau^{-2}}\left(\text { for } 0<t<t_{1}\right), \quad P(t)=0\left(\text { for } t \leqslant 0 \text { or } t_{1} \leqslant t\right)
$$


was set, calculated using approximated dependence of the laser radiation intensity

$$
I_{1}(t)=I_{0} 16^{-\left(t-t_{0}\right)^{2} \tau^{-2}}
$$

and the scaling relation, which is formulated for the range $4.3<I_{0} \leqslant 1000 \mathrm{TW} / \mathrm{cm}^{2}[22,23]$ :

$$
P_{\mathrm{a}}=P_{\mathrm{a} 0}\left(\lambda_{I 0} I_{0} / \lambda\right)^{2 / 3}\left[A_{\mathrm{u}} /(2 Z)\right]^{3 / 16},
$$

where $P_{\mathrm{a} 0}=1.2 \mathrm{TPa} ; \lambda_{I 0}=10^{-2} \mu \mathrm{m} \mathrm{cm}^{2} / \mathrm{TW} ; A_{\mathrm{u}}$ and $Z$ are the atomic mass (u) and the atomic number of the target material respectively, $A_{\mathrm{u}}=26.98154$ and $Z=13$ for aluminum. On the rear side of the target, pressure was set equal to zero.

\section{SIMULATION RESULTS}

The simulation was performed for the case of loading pressure pulse (31) with the magnitude $P_{\mathrm{a}}=516 \mathrm{GPa}$ according to equation (33); $t_{0}=123 \mathrm{ps}, t_{1}=246 \mathrm{ps}$.

Figure 1 illustrates the change in pressure during the propagation of compression and rarefaction waves through the aluminum target. In figure $1(a)$, one can see that the rarefaction wave follows the shock wave while both move towards the rear side of the target. After the shock wave has reached the rear side, one more rarefaction wave begins to move backward [see figure $1(b)$ ]. When these two rarefaction waves meet, a spalling phenomenon occurs.

Figure 2 shows the calculated pressure and density histories in three planes, which correspond to the initial distances from the rear side of the target 9, 10 and $11 \mu \mathrm{m}$.

The curves shown in figure 2(a) allow one to estimate the maximum possible tensile stress $\sigma_{\max }$ in the sample in the spall plane. The absolute value of the pressure at the minimum on the curve for the plane where the spallation occurred in the experiment is this maximum possible tensile stress. The difference in values in two adjacent planes (for which the initial position differs from the initial position of the spall plane by the value of the error in determining the spall depth), divided in half, gives the average error in determining the maximum possible tensile stress in the sample.

The calculated curves shown in figure $2(b)$ allow one to estimate the maximum strain rate $\rho_{0} \mathrm{~d} V / \mathrm{d} t=-\rho_{0} \rho^{-2} \mathrm{~d} \rho / \mathrm{d} t$ in the spall plane at the stage of tension at negative pressures. Here, $V=\rho^{-1}$ is the specific volume. Starting from the point of zero pressure, when the sample is stretched, the strain rate decreases monotonically to zero at the point of minimum pressure. Then, with time, the tensile stress decreases, and the strain rate becomes negative (i.e., the density increases with pressure).

In the case under consideration, $\sigma_{\max } \pm \Delta \sigma_{\max } \approx 7.2 \pm 0.5 \mathrm{GPa}, \rho_{0} \mathrm{~d} V / \mathrm{d} t \pm \Delta\left(\rho_{0} \mathrm{~d} V / \mathrm{d} t\right) \approx$ $0.22 \pm 0.01 \mathrm{~ns}^{-1}$ for aluminum. 

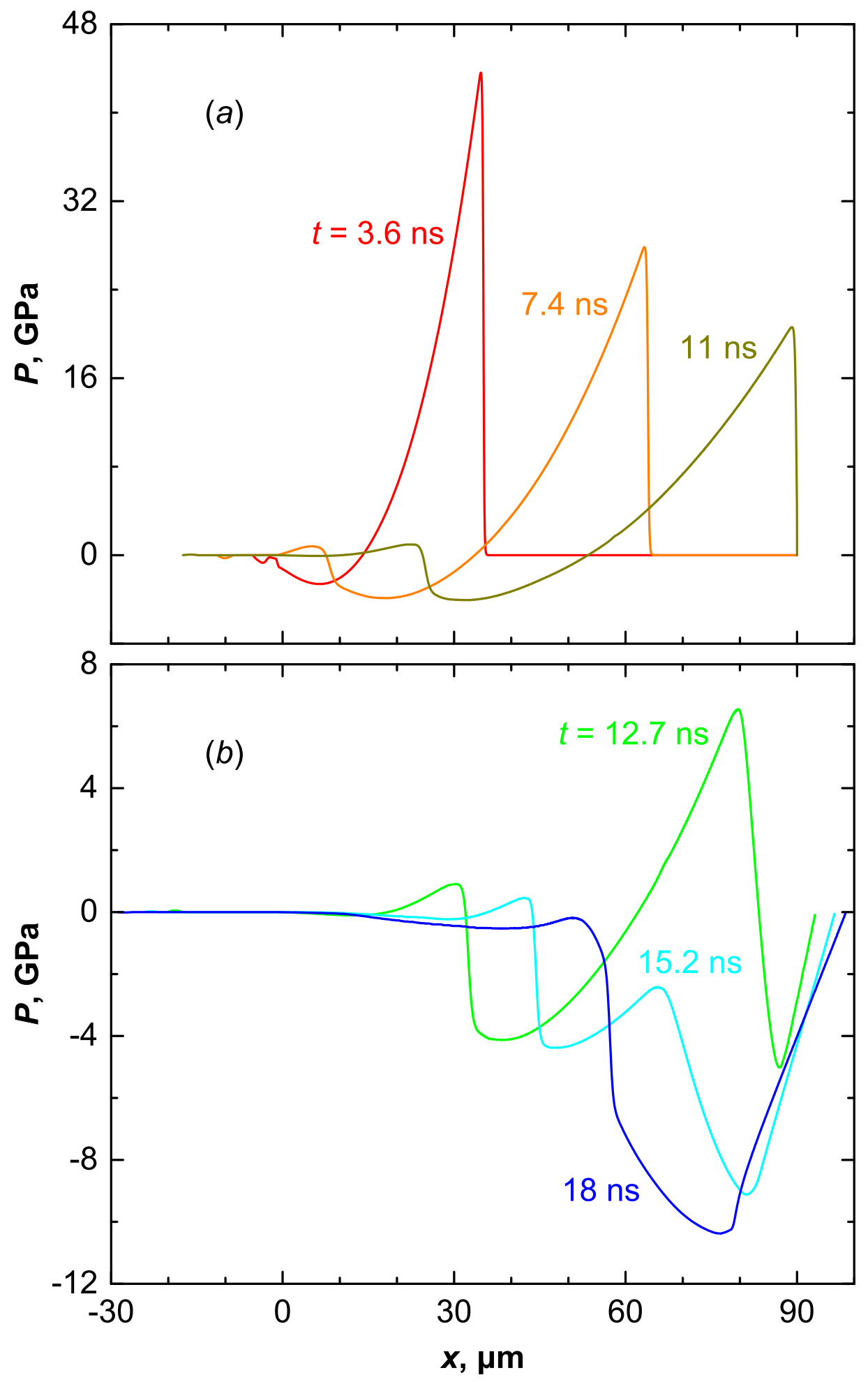

Figure 1: Pressure in the target at $t=3.6,7.4,11(a), 12.7,15.2$ and $18 \mathrm{~ns}(b)$ along the coordinate axis $x$, which is perpendicular to the irradiated surface, with the origin at the point of the initial position of this surface before the experiment. 


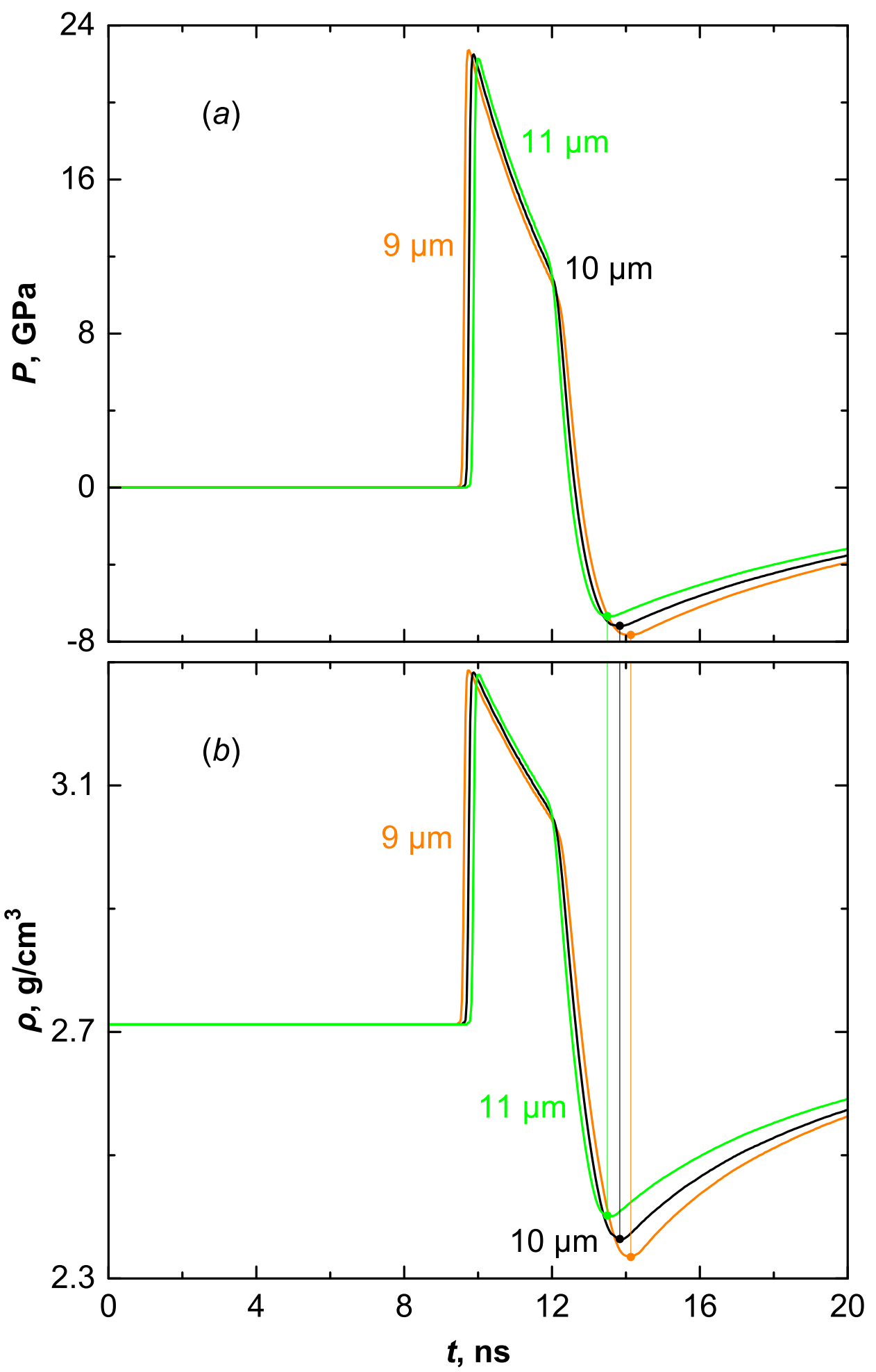

Figure 2: Pressure $(a)$ and density $(b)$ histories in three planes that correspond to the initial distances from the back of the target 9, 10 and $11 \mu \mathrm{m}$. The thin vertical lines correspond to the moments of reaching the maximum tensile stress (negative pressure). 


\section{CONCLUSIONS}

Thus, in a laboratory experiment on irradiating a $90-\mu \mathrm{m}$-thick aluminum plate with a $70 \mathrm{ps}$ laser pulse with a maximum intensity of $14.7 \mathrm{TW} / \mathrm{cm}^{2}$, a spall was obtained at a distance of $10 \pm 1 \mu \mathrm{m}$ from the rear surface of the target. A hydrodynamic model has been developed for the propagation and interaction of shock and release waves in a target under such a pulsed action. As a result of the calculation using the developed model, the maximum possible tensile stress in the sample in the spall plane is determined as $7.2 \pm 0.5 \mathrm{GPa}$ and the maximum strain rate at the stage of tension at negative pressures is determined as $0.22 \pm 0.01 \mathrm{~ns}^{-1}$.

Acknowledgments: The authors dedicate this article to the memory of their teacher, colleague and friend Igor Kornelievich Krasyuk (07.03.1942-26.05.2020).

This work is financially supported by the Russian Foundation for Basic Research (project No. 20-02-00683).

The paper is based on the proceedings of the XXXVI International Conference on Interaction of Intense Energy Fluxes with Matter, Elbrus, the Kabardino-Balkar Republic of the Russian Federation, March 1 to 6, 2021.

\section{REFERENCES}

[1] S. I. Anisimov, A. M. Prokhorov, and V. E. Fortov, "The use of powerful lasers for the study of matter under superhigh pressures", Usp. Fiz. Nauk, 142(3), 395-434 (1984).

[2] V. E. Fortov, D. Batani, A. V. Kilpio, I. K. Krasyuk, I. V. Lomonosov, P. P. Pashinin, E. V. Shashkov, A. Yu. Semenov, and V. I. Vovchenko, "The spall strength limit of matter at ultrahigh strain rates induced by laser shock waves", Laser Part. Beams, 20(2), 317-320 (2002).

[3] D. Batani, V. I. Vovchenko, G. I. Kanel, A. V. Kilpio, I. K. Krasyuk, I. V. Lomonosov, P. P. Pashinin, A. Yu. Semenov, V.E. Fortov, and E. V. Shashkov, "Mechanical properties of a material at ultrahigh strain rates induced by a laser shock wave", Dokl. Phys., 48(3), 123-125 (2003).

[4] M. A. Barrios, D. G. Hicks, T. R. Boehly, D. E. Fratanduono, J. H. Eggert, P. M. Celliers, G. Collins, and D. D. Meyerhofer, "High-precision measurements of the equation of state of hydrocarbons at 1-10 Mbar using laser-driven shock waves", Phys. Plasmas, 17(5), 056307 (2010).

[5] S. I. Ashitkov, M. B. Agranat, G. I. Kanel', P. S. Komarov, and V.E. Fortov, "Behavior of aluminum near an ultimate theoretical strength in experiments with femtosecond laser pulses", JETP Lett., 92(8), 516-520 (2010).

[6] R. E. Rudd, T. C. Germann, B. A. Remington, and J. S. Wark, "Metal deformation and phase transitions at extremely high strain rates", MRS Bull., 35(12), 999-1006 (2010).

[7] S. I. Ashitkov, P. S. Komarov, A. V. Ovchinnikov, E. V. Struleva, and M. B. Agranat, "Deformation dynamics and spallation strength of aluminium under a single-pulse action of a femtosecond laser", Quantum Electron., 43(3), 242-245 (2013).

[8] I. K. Krasyuk, P. P. Pashinin, A. Yu. Semenov, K. V. Khishchenko, and V.E. Fortov, "Study of extreme states of matter at high energy densities and high strain rates with powerful lasers", Laser Phys., 26(9), 094001 (2016).

[9] C. A. McCoy, S. X. Hu, M. C. Marshall, D. N. Polsin, D. E. Fratanduono, Y. H. Ding, P. M. Celliers, T. R. Boehly, and D. D. Meyerhofer, "Measurement of the sound velocity and Grüneisen parameter of polystyrene at inertial confinement fusion conditions", Phys. Rev. B, 102(18), 184102 (2020).

[10] S. A. Abrosimov, A. Bazhulin, V. Voronov, I. Krasyuk, P. Pashinin, A. Semenov, I. Stuchebryukhov, and K. V. Khishchenko, "Study of mechanical properties of aluminum, AMg6M alloy, and poly- 
methyl methacrylate at high strain rates under the action of picosecond laser radiation", Dokl. Phys., 57(2), 64-66 (2012).

[11] S. A. Abrosimov, A. P. Bazhulin, V. V. Voronov, A. Geras'kin, I. Krasyuk, P. Pashinin, A. Semenov, I. A. Stuchebryukhov, K. V. Khishchenko, and V. E. Fortov, "Specific features of the behaviour of targets under negative pressures created by a picosecond laser pulse", Quantum Electron., 43(3), 246-251 (2013).

[12] A. A. Samokhin, V. I. Mazhukin, M. M. Demin, A. V. Shapranov, and A. E. Zubko, "On critical parameters manifestations during nanosecond laser ablation of metals", Math. Montis., 43, 38-48 (2018).

[13] S. Yu. Gus'kov, I. K. Krasyuk, A. Yu. Semenov, I. A. Stuchebryukhov, and K. V. Khishchenko, "Extraction of the shock adiabat of metals from the decay characteristics of a shock wave in a laser experiment", JETP Lett., 109(8), 516-520 (2019).

[14] K. K. Maevskii, "Thermodynamic parameters of mixtures with silicon nitride under shock-wave loading", Math. Montis., 45, 52-59 (2019).

[15] A. A. Samokhin, P. A. Pivovarov, and A. L. Galkin, "Modeling of transducer calibration for pressure measurement in nanosecond laser ablation", Math. Montis., 48, 58-69 (2020).

[16] V. I. Mazhukin, M. M. Demin, A. V. Shapranov, and A. V. Mazhukin, "Role of electron pressure in the problem of femtosecond laser action on metals", Appl. Surf. Sci., 530, 147227 (2020).

[17] A. G. Kulikovskii, N. V. Pogorelov, and A. Yu. Semenov, Mathematical Aspects of Numerical Solution of Hyperbolic Systems, Monographs and Surveys in Pure and Applied Mathematics, Vol. 118, Boca Raton, FL: Chapman \& Hall/CRC, (2001).

[18] K. V. Khishchenko, "The equation of state for magnesium at high pressures", Tech. Phys. Lett., 30(10), 829-831 (2004).

[19] K. V. Khishchenko, "Equation of state for tungsten over a wide range of densities and internal energies", J. Phys.: Conf. Ser., 653, 012081 (2015).

[20] K. V. Khishchenko, "Equation of state for titanium at high energy densities", J. Phys.: Conf. Ser., 774, 012001 (2016).

[21] R. Courant, E. Isaacson, and M. Rees, "On the solution of nonlinear hyperbolic differential equations by finite differences", Commun. Pure Appl. Math., 5(3), 243-255 (1952).

[22] V. I. Vovchenko, I. K. Krasyuk, P. P. Pashinin, and A. Yu. Semenov, "Wide-range ablation pressure scaling law for laser experiments", Dokl. Akad. Nauk, 338(3), 322-324 (1994).

[23] I. K. Krasyuk, A. Yu. Semenov, I. A. Stuchebryukhov, and K. V. Khishchenko, "Experimental verification of the ablation pressure dependence upon the laser intensity at pulsed irradiation of metals", J. Phys.: Conf. Ser., 774, 012110 (2016).

Received December 20, 2020 